\section{LANDMINES DETECTION TECHNOLOGIES: A COMPARATIVE STUDY}

\section{H. Kashan", O. Zahran**, M. El-kordly**, S. M. Elaraby* And F. E. Albd El-samie*}

* Engineering Department, Nuclear Research Center, Atomic Energy Authority, Egypt

** Department of Electronics and Communications, Faculty of Electronic Engineering, Menoufia University, Egypt.

$$
\begin{array}{r}
\text { E-mails: hany_kasban@yahoo.com } \\
\text { osama_zahran@hotmail.com } \\
\text { fathi_sayed@yahoo.com }
\end{array}
$$

\section{Abstract}

Many techniques can be used in landmine detection. The performance of each technique depends on the nature of the contaminated soil and the type of the landmine buried. This paper offers a survey of the most recent advances in landmine detection techniques such as biological, electromagnetic, optical, nuclear, acoustic, and mechanical. Biological detection techniques include the use of dogs, rats, bees, plants and bacteria, while electromagnetic detection techniques include metal detector (MD), ground penetrating radar (GPR), microwave radiometry (MWR) and millimeter wave radiometry (MMWR) Optical detection techniques, however, include visible light and light detection and ranging (LIDAR), as opposed to nuclear detection techniques which include nuclear quadrople resonance (NQR) and the neutron based method. Yet, acoustic detection techniques include the use of ultrasound method and the acoustic to seismic (A/S). By contrast, mechanical detection techniques include mine clearing machines. These techniques are briefly described and their relative merits and drawbacks are highlighted and compared. A comparison among those techniques is also presented in order to show the ideal working conditions for, and the inherent challenges involved in the use of, each technique. Other, equally significant, factors of comparison will also be included in this study, such as cost, complexity, speed, safety, false alarm rate and influence of environmental conditions.

\section{Introduction}

At least, 70 countries are defined as suffering from the existence of millions of antipersonnel (AP) and antitank (AT) landmines in their lands. These landmines have indefinite lives, and may still cause horrific personal injuries and economic dislocation for decades after a war was finished. Therefore, there is a growing demand in these countries for reliable landmine detection systems. Landmines are small explosive objects that are designed to kill, maim, wound, or otherwise incapacitate humans, and are designed to destroy or damage vehicles. Each landmine consists of three main components; the case that may be made of metal, wood, plastic or a mixture of these materials, the explosive material that may be Trinotrotoluene (TNT), Royal Demolition Explosive (RDX) or a mixture of both and the ignitor which is responsible of exploding the mine. There are more than 100 million AP and AT buried landmines covering more than 200,000 square kilometers of the world's surface (1) The International Campaign to Ban Landmines (ICBL), reported that in 2006 there were more than 5751 mine casualties in 68 countries (1). The production cost of landmine is very low (around $3 \$$ to $30 \$$ ) but the detection and removal cost is still very high (around $300 \$$ to $1000 \$$ ) (2).To solve this problem, many techniques have been designed and developed for detecting and clearing these buried landmines. This paper is organized as follows:

Section 2 presents the biological techniques.

Section 3 presents the electromagnetic techniques.

Section 4 presents the optical techniques.

Section 5 discusses the nuclear techniques.

Section 6 presents the acoustic techniques.

Section 7 presents the mechanical techniques. S ection 8 presents a comparison among landmine detection techniques.

Finally, section 9 gives the concluding remarks. 


\section{Biological Detection}

Dogs: Dogs can be used for landmines detection by use of their highly developed senses (3). The main advantage of this method is that dogs can smell explosives buried at a depth of $60 \mathrm{~cm}$ (4).The main drawback of this method is that the performance varies widely depending on the individual dog, and its training duration and methods.

Rodents: Rats are trained using food rewards to signal the presence of explosives by scratching the ground surface with their feet (5). The advantage of this method is that rodents are lighter and easy to educate, transport and feed. The limitations of this method is that rats can only work under limited weather conditions.

Honey bees: Bees are trained by feeding them on points where the soil has been impregnated with explosive chemicals like TNT. Based on bees' keen sense of smell, they have the ability to connect the smell of explosives with food (6).The advantage of this method is that it provides greater accuracy and a higher clearance rate. The disadvantage of this method is the need for a mechanism to transport bees after detection process.

Plants: The mustard arabidopsis thaliana is one of the most widlly studied plants in the world. This plant normally turns red under harsh conditions. Using a combination of natural mutations and genetic manipulations methods, a strain is developed that only changes color in response to the nitrous oxide that leaks from landmines and other explosives (7). The main advantage of this method is that the plants could be thrown from an aircraft. So it can cover a large area in a short time. The disadvantage of this method is the high false alarm rate.

Bacteria: Bacterial mine detection process involves spraying an engineered strain of bacteria on the mine affected area, possibly using an airborne system (4). The bacteria would be allowed to grow for several hours. It, then, gives fluoresce colour under ultraviolet light in the presence of the TNT material. The main advantages of this method are quicker results, larger coverage and shorter periods of time. The limitations of this method are that the bacteria are highly sensitive to environmental conditions. Also, no strain of bacteria is capable of detecting RDX explosive.

\section{Electromagnetic Detection}

MD: The MD system is based on the electromagnetic induction (EMI), where it is composed of a primary coil (transmitter) and one or more secondary coils (receiver). A time-varying current in the transmitter coil generates a low frequency in electromagnetic field, which induces electric currents in nearby metallic objects. These eddy currents in turn induce a time varying current in the receiver coil, which is amplified and processed to provide an indication (acoustic signal for example) for the presence of mines (8). The main advantages of this technique are its ability to detect and see metal parts of radius less than $1 \mathrm{~cm}$ to a depth of $50 \mathrm{~cm}$ (4), its low cost, ease of use, and reliability in all weather and soil moisture conditions. The limitations of this technique are that it cannot detect landmines with a very low metal content and the newer plastic mines. These plastic mines give a high false alarm rate.

GPR: The GPR operates by transmitting an electromagnetic signal into the soil and detecting the reflected signal at the receiver $(5,8)$. The transmitter emits a pulse wave or a continuous wave at a given frequency. The receiver collects the waves backscattered by discontinuities in permittivity. Discontinuities can be provoked by both the buried objects like landmines (useful signal) and the natural discontinuities in the soil (clutter). The main advantages of this technique are its ability to detect plastic objects buried in the ground, its ability to provide target depth information, and its low false alarm rate. The limitations of this technique are that microwaves are strongly attenuated by certain types of conductive soils such as clay and the difficulty to detect plastic landmines in a dry soil. 
MWR: This technique is based on the transmission of short radio and microwave radiation pulses from an antenna into the ground and measuring the time for reflections returning back to the same antenna. Reflections occur at the boundaries between materials of different dielectric constants that are orthogonal to the incident radiation (6). The main advantage of this method is its ability to provide information about object depth. The limitations of this method are that its disability of giving a good results in a wet soil and the effect of radio frequency interference.

MMWR: A novel active MMW scanning system was developed for the detection of buried landmines. It is a hyperspectral system that collects images at different MMW frequencies using a vector network analyzer collecting the backscattered MMW radiation from the buried sample (9). A multivariate Principal Components Analysis (PCA) method is applied to extract useful information from these images. The main advantage of this method is its ability to detect metallic objects buried under 3 inches of dry sand (10). The limitation of this method is that the emissions are typically weak.

\section{Optical Detection}

Visible light detection: Visible light detection involves capturing light waves of visible wavelights using an image formation optical system, such as a camera (10). A visual imager gathers a beam of light from an object, points and transforms it into a beam that converges toward, or diverges from, other points on a focal plane, thereby producing an image. The main advantages of this technique are that the detection process can be achieved from an airborne platform enabling large areas to be scanned in a relatively short time. It can detect both metallic and nonmetallic landmines. The limitations of this method are that it can be easily blocked by camouflage or foliage. It can be used only on flat land with little vegetation
LIDAR detection: The LIDAR is an optical technology that works in the visible and infrared regions of the electromagnetic spectrum. LIDAR send out pulses of coherent radiation, a fraction of which is reflected back by surface-laid objects (10). LIDAR sensors measure both the traveling time of the reflected pulses and the difference between the transmitted and the reflected energy, which are used to calculate the distance to the target and its general reflectivity or absorption. The main advantages of this method are its safety and ability to scan large areas in a short time. The limitations of this method are its inability to work well in moderately to highly vegetated areas and its high cost.

\section{Nuclear Detection}

NQR detection: In this method the observed frequencies depend on the interaction between the electric quadrupole moment of the nucleus and the electric field gradient generated at the nuclear site by external charges. All common high explosives contain a quadrupolar nucleus which generates three sets of resonance frequencies, providing an unequivocal method of detecting and identifying the explosive (11). The main advantage of this method is that it has a very low false alarm rate. The limitation of this method is that the detection of TNT is much harder than RDX.

Neutron-based detection: In this Method, a continuous or pulsed neutron source that emits bursts of neutrons into the ground is used. A detector is used to characterize the outgoing radiations, which is predominantly composed of gamma rays that result from interactions of neutrons with the soil and the substances such as explosives (3). This Method works on the principle that the nuclei of the chemical elements in the explosive and /or in the landmine case can be bombarded by a penetrating nuclear radiation(12). The main advantage of this method is that the pulsed systems allow the use of timing information which can be useful for reducing the influence of the background radiation from neutron interactions with the 
soil. The limitations of this method are the high cost, the high power consumption and the radiation hazards

\section{Acoustic Detection}

Ultrasound detection: Ultrasound detection consists of emission of a sound wave with a frequency higher than 20 $\mathrm{kHz}$ into a medium. This sound wave will be reflected on the boundaries between materials with different acoustical properties (4). The main advantage of this method is that its high capacity for good penetration through very wet and heavy ground such as clay. Ultrasound is very good and almost the only means used for underwater mine detection. The limitation of this method is the typical problems met at the interface of the air and the ground.

A/S detection: The $\mathrm{A} / \mathrm{S}$ method is intended for the detection of landmines by vibrating them with acoustic or seismic waves that are generated and received by acoustic or seismic transducers, respectively. These detection methods are based on the mechanical properties that can differentiate between the acoustic response of mines and that of other objects buried in the ground (13). The main advantages of this method are its high sensitivity to detect $\mathrm{AP}$ and AT mines as well as its low false alarm rate. The limitation of this method is its low detection speed. In the presence of heavy vegetation on the ground, the detection speed is limited by measurement technologies and the heavily covered grassy surfaces, particularly those with dead vegetation not directly rooted into the soil.

\section{Mechanical Detection}

Mine clearing machines: When there is not a lot of time for an army to clear a minefield, certain machines are often emploied to roll through and create a safe path. Military forces employ several kinds of mine clearing machines to clear out or detonate mines (4). Many are available. New machines are remote controlled, which minimizes the risk to personnel. The main advantages of this method are its quickness and efficiency and minimal chance of injuries during the detection. The limitation of this method is that it leaves the area virtually destroyed.

\section{8.comparisons Among Landmine Detection Techniques}

Based on the above discussions, we can, perhaps, surmise that the performance of each landmine detection technique depends on the nature of the contaminated soil and the type(s) of the buried landmines. A comparison among landmine detection techniques to show the ideal conditions and challenges for each technique is shown in Table I. Furthermore, a comparison between landmine detection techniques from the points of view of cost, complexity, speed, safety, false alarm rate and the influence of the environmental conditions is shown in Table II. Comparisons show that dogs, rodents, bees, $\mathrm{MD}$, EIT, light and prodders are cheaper detection techniques, but also slower in process, and higher in risk for lives. Bacterial, GPR, NQR and A/S, by contrast, give low false-alarm rates, and can be considered as reliable landmine detection techniques. 
Table I Ideal conditions and challenges of landmine detection techniques

\begin{tabular}{|c|c|c|c|c|c|c|c|}
\hline \multirow[t]{2}{*}{ Technique } & \multirow[t]{2}{*}{ Sensor } & \multicolumn{3}{|c|}{ Ideal conditions } & \multicolumn{3}{|c|}{ Challenges } \\
\hline & & Mine case & $\begin{array}{c}\text { Explosive } \\
\text { material }\end{array}$ & $\begin{array}{c}\text { Nature of } \\
\text { Soil }\end{array}$ & $\begin{array}{l}\text { Mine } \\
\text { case }\end{array}$ & $\begin{array}{c}\text { Explosive } \\
\text { material }\end{array}$ & Nature of Soil \\
\hline \multirow{5}{*}{$\begin{array}{l}\text { Biological } \\
\text { detection }\end{array}$} & Dogs & All & \multirow{3}{*}{$\begin{array}{l}\text { One types which } \\
\text { they are trained } \\
\text { on it }\end{array}$} & All & Non & \multirow{3}{*}{$\begin{array}{c}\text { Any types } \\
\text { which they are } \\
\text { not trained on it }\end{array}$} & Non \\
\hline & Rodents & All & & All & Non & & Non \\
\hline & Bees & All & & All & Non & & Non \\
\hline & Plants & All & TNT & Wet clay & Non & RDX & Rocks \\
\hline & Bacteria & All & TNT & All & Non & RDX & Non \\
\hline \multirow{4}{*}{$\begin{array}{c}\text { Electro } \\
\text { magnetic } \\
\text { detection }\end{array}$} & MD & Metallic & All & All & $\begin{array}{c}\text { Plastic, } \\
\text { wood }\end{array}$ & Non & Non \\
\hline & GPR & Plastic & All & Dry & Non & Non & Wet clay \\
\hline & MWR & Metallic & All & Dry & Non & Non & Wet \\
\hline & MMWR & Metallic & All & Dry & Non & Non & Non \\
\hline \multirow{2}{*}{$\begin{array}{c}\text { Optical } \\
\text { detection }\end{array}$} & V. light & ALL & ALL & All (flat) & Non & Non & Blocked \\
\hline & Lidar & All & All & All & Non & Non & Vegetations \\
\hline \multirow{2}{*}{$\begin{array}{l}\text { Nuclear } \\
\text { detection }\end{array}$} & NQR & $\begin{array}{c}\text { Plastic, } \\
\text { wood }\end{array}$ & $\mathrm{RDX}$ & ALL & Metal & TNT & Non \\
\hline & Neutron & All & All & All & Non & Non & Non \\
\hline \multirow{2}{*}{$\begin{array}{l}\text { Acoustic } \\
\text { detection }\end{array}$} & $\mathrm{A} / \mathrm{S}$ & All & All & wet & Non & Non & Vegetations \\
\hline & Ultrasound & All & All & Wet & Non & Non & Dry \\
\hline $\begin{array}{l}\text { Mechanical } \\
\text { detection }\end{array}$ & $\begin{array}{l}\text { Clearing } \\
\text { machines }\end{array}$ & All & All & All & Non & Non & Non \\
\hline
\end{tabular}

Table II. Comparison between landmine detection techniques

\begin{tabular}{|c|c|c|c|c|c|c|c|}
\hline Technique & Sensor & Complexity & Cost & speed & Safety & $\begin{array}{c}\text { Environment } \\
\text { effect }\end{array}$ & $\begin{array}{l}\text { False } \\
\text { alarm }\end{array}$ \\
\hline \multirow{5}{*}{$\begin{array}{l}\text { Biological } \\
\text { detection }\end{array}$} & Dogs & Low & Medium & Medium & Medium & Medium & Medium \\
\hline & Rodents & Low & Low & Low & High & High & High \\
\hline & Bees & Low & Medium & Low & High & High & High \\
\hline & Plants & Medium & Medium & Low & High & High & High \\
\hline & Bacteria & Medium & Medium & High & Low & High & Low \\
\hline \multirow{4}{*}{$\begin{array}{l}\text { Electro } \\
\text { magnetic } \\
\text { detection }\end{array}$} & MD & Low & Low & Low & High & Low & High \\
\hline & GPR & Medium & High & Medium & High & Medium & Low \\
\hline & MWR & Medium & Medium & Low & High & Medium & Medium \\
\hline & MMWR & High & Medium & Low & High & Medium & Medium \\
\hline \multirow{2}{*}{$\begin{array}{c}\text { Optical } \\
\text { detection }\end{array}$} & V. light & Low & Low & Medium & High & High & High \\
\hline & Lidar & High & High & Medium & High & Low & Medium \\
\hline \multirow{2}{*}{$\begin{array}{l}\text { Nuclear } \\
\text { detection }\end{array}$} & NQR & High & Medium & Medium & Medium & High & Low \\
\hline & Neutron & High & High & High & Low & Low & Medium \\
\hline \multirow{2}{*}{$\begin{array}{l}\text { Acoustic } \\
\text { detection }\end{array}$} & $\mathrm{A} / \mathrm{S}$ & Medium & High & Medium & High & Low & Low \\
\hline & Ultrasound & Medium & Medium & Low & High & Medium & High \\
\hline $\begin{array}{l}\text { Mechanical } \\
\text { detection }\end{array}$ & $\begin{array}{l}\text { Clearing } \\
\text { machines }\end{array}$ & Medium & Low & High & Low & Low & High \\
\hline
\end{tabular}




\section{Conclusion}

There are many techniques that can be used in buried landmine detection. The performance of each technique depends on the nature of the contaminated soil and the type of buried mines. With respect to the nature of the contaminated soil; use of dogs, rodents, bees, bacteria, MD, MWR, NQR and neutron techniques can be beneficial regardless of the type of soil involved. Use of plants, by comparison, give good results in wet clay soils and can't be beneficial in rocky soils too. Use of GPR and MWR give good results in dry soils, but can't be beneficial in the wet ones. Use of ultrasound gives good results in wet soils, and can't be used in dry ones. Use of LIDAR and the A/S can't be beneficial in vegetations soils. With respect to the mine case; most detection techniques can be used for all types except the MD, which can only be used for metal-cased mines. The NQR technique, however, can not be used for the metal case mines. With respect to the explosive material; most detection techniques can be used for all types of explosives, except the use of plants and bacteria which can only be used for TNT mines. Also the NQR technique can only be used for RDX mines.

\section{References}

1. International Campaign to Ban Landmines, "Landmine Monitor Report", (2007).

2. J. k. Paik, C. P. Lee and M. A. Abidi, Int. J. Sens Tech. Appl., 3 (2002) 153.

3. S. Sen and R. L Woodfin, Am. Phys. Soc., 31 (2002)

4. C. P Gooneratne, S. C. Mukhopahyay and G. S. Gupta, 2nd International Conference on Autonomous Robots and Agents, Palmerston North, New Zealand, 2004. http://wwwist.massey.ac.nz/conferences/ icara2004/files/Papers/Paper70_ICARA2004 401407. pdf

5. M Acheroy, and I. D Bosch, Detection of Explosives and Landmines, 66(2002) 39.

6. S Anitei and k. Kezic, Oakridge National Laboratory, 2007 www.ornl.gov/info/news/pulse/pulse_v30_99.pdf

7. A. A . Berhe, :, Land degradation and development,. 18(2007)1.

8. Y. Liao, L.W Nolte, and L. M. Collins, IEEE Trans.on Geo. and remote sensing, 45 (2007) 398.

9. T. W Bosq, M. L Alonso, G. D Boreman and D Dillery , Proc. SPIE, 6217 (2006)

10. J MacDonald and J.R. Lockwood, Alternatives for Landmine Detection, online book,_0-8330-3301-8, 2003

11. A. Jakobsson, M. Mossberg and A. S. Smith, IEEE Trans. signal processing, 54 (2006) 1610

12. Valkovic, V and Antonic, D .: Humanitarian Demining, Second Australian American Joint Conference on the Technologies of Mines and Mine Countermeasures, Sydney, Australia, 2001

13. J. M. Sabatier, N. Xiang, A. G. Petculescu, and M. Bradley, International Conference on Requirements and Technologies for the Detection of Landmines, Brussels, Belgium, 2003 